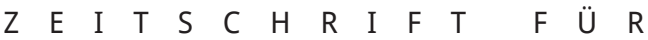

$\begin{array}{lllllllllllllllllllll}V & E & R & W & A & L & T & U & N & G & S & G & E & S & C & H & I & C & H & T & E\end{array}$

$\begin{array}{lllllllll}B & A & N & D & 3 & 2 & 0 & 1 & 8\end{array}$

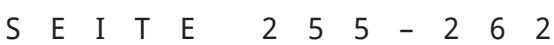

D O I : $10.2478 / \mathrm{ADH}$ I - $2018-0040$

\title{
Wieder gelesen: Fritz Morstein Marx, "Das Dilemma des Verwaltungsmannes« (1965)
}

\section{MARGRIT SECKELMANN}

Ein Themenheft $\mathrm{zu} »$ Verwaltung und Emotionen « - ist Fritz Morstein Marx (1900-1969) ${ }^{1}$ als Autor da wirklich der Richtige? Er, der sein Leben lang die Dinge mit sich selbst ausmachte und sogar seine eigene Familie über die Gründe für seine Remigration nach Deutschland zeitlebens im Ungewissen ${ }^{2}$ ließ?

Während er seine eigenen Gefühle also eher hinter einer Form distanzierenden Humors verbarg, der sich mit einer großen Menschenfreundlichkeit verband, ${ }^{3}$ beschrieb er mit dem gleichen Humor liebevoll den »Verwaltungsmann«, dem seine Sympathie galt. ${ }^{4}$ Hier zeichnete er verwaltungsanthropologische Vignetten, die noch heute nichts an Aktualität eingebüßt haben ${ }^{5}$ und die man vielleicht eher beim frühen Niklas Luhmann $^{6}$ vermuten würde. Er mag ihn in dieser Hinsicht vielleicht sogar ein wenig beeinflusst haben, schließlich arbeiteten beide zu der Zeit in Speyer auf demselben Campus. ${ }^{7}$

Daher lohnt es sich, auf Morstein Marx' Schrift »Das Dilemma des Verwaltungsmannes« näher einzugehen, die im Jahr 1965 bei Duncker und Humblot in der Schriftenreihe der Hochschule Speyer erschien und die auf drei zuvor in den USA veröffentlichte Aufsätze zurückgeht. $^{8}$

\section{Grundsätzliches}

In diesem Band beschäftigt sich Morstein Marx mit dem "Verwalten «, ${ }^{9}$ das sowohl als Organisations- wie als Tätigkeitsbegriff verstanden wird. Stärker noch als beim Wort "Verwaltung«, das auch beide Sinndimensionen erfasst, wird aber hier "die Blickrichtung des Handelnden selbst «, also des Amtswalters, zugrunde gelegt. ${ }^{10}$ Es geht Morstein Marx um die "persönliche Auseinandersetzung " des "Verwaltungsmannes» mit »den gegebenen Umständen «, ${ }^{11}$ allerdings weniger im Sinne sozialempirischer Erhebungen als mit Mitteln einer Art Verwaltungsanthropologie. Für eine sozialempirische Erhebung zur "Politischen Kultur" hätten 1963 Gabriel Almond und Sidney Verba einen Ansatz geboten. ${ }^{12}$ Morstein Marx bezieht sich aber stärker auf die Überlegungen Luther Gulicks, der die Forschungsrichtung des "Behavioralismus" (mit-) begründet hatte, also die Erklärung des Verhaltens der Verwaltung(smitarbeiter) durch behavioristische Verhaltensannahmen. ${ }^{13}$ Auch wenn Morstein Marx sich um 1965 vorsichtig von den etwas holzschnittartigen Vorstellungen seines akademischen Förderers Gulick zu lösen begann, ${ }^{14}$ war diese Loslösung deutlich weniger 
deutlich und gleichsam organischer als beispielsweise bei Herbert A. Simon, der Gulicks nahezu überzeitliche Annahmen von sder Verwaltung spöttisch als "Sprichwörter « charakterisierte..$^{15}$ Was Morstein Marx jedoch mit Almond, Verba und Simon verbindet, ist sein Abheben auf die kulturellen Bedingtheiten des Verwaltens. Mit Carl Joachim Friedrich ${ }^{16}$ gehörte Morstein Marx zu den Vertretern eines "neuen Realismus" der Verwaltungswissenschaft. ${ }^{17}$ Anders als Woodrow Wilson, ${ }^{18}$ der zwischen Regierung und Verwaltung strikt trennte und in dessen Tradition Gulick stand, stellte Morstein Marx insbesondere auf die (auch kulturelle) Einbettung von Verwaltung ab und führte insoweit Regierung und Verwaltung wieder stärker zusammen.

\section{Das Dilemma des Verwaltungsmannes}

\section{Aufbau}

Die hier vorzustellende Schrift ist in neun Kapitel gegliedert. Diese beziehen sich eher "verwaltungsübergreifend « auf das, was zumindest in den westlichen Demokratien die Themen der Verortung von Verwaltung waren: 1. "Entscheidung und Apparat«, 2. »Umwelt und Verwaltung «, 3. "Vorrichtungen und Verhalten«, 4. "Status und Funktion«, 5. "Bereiche des Verwaltungshandelns«, 6. "Zwischen Wollen und Müssen" (mit der Sowjetorganisation als einer Art Negativfolie), 7. "Bestand und Wandel«, 8. "Sachgerechtigkeit und Rechtmäßigkeit" und gleichsam als Summe der Überlegungen zuvor - 9 . "Zwischen Stehen und Fallen«.

Die zentrale Aussage ist diejenige: Verwalten muss auch immer eine gestalterische Komponente innehaben. Tut es das nicht, kann eine Beschränkung auf den bloßen Normvollzug langfristig zu Politikverdrossenheit führen, die der auf das Gemeinwohl verpflichtete "Verwaltungsmann" eigentlich nicht dulden kann. Jedoch droht der »Verwaltungsmann« bei einer gestalterischen Politikausführung seine Kompetenzen zu überschreiten, weswegen er Nachteile erleiden könnte. Diese beziehen sich zum einen auf dienstrechtliche Konsequenzen, zum anderen aber auch auf die Zeit, von Herbert A. Simons "administrative man ${ }^{19}$ ) aufwenden muss, um sich einen Überblick über den Sachverhalt zu verschaffen. Das titelgebende Dilemma besteht darin, dass sich ein verantwortungsbewusster Verwaltungsmitarbeiter oder eine verantwortungsbewusste Verwaltungsmitarbeiterin insoweit immer mehr Arbeit schafft, wenn er oder sie sich nicht hinter der bloßen Ausführung von Weisungen verschanzen möchte. Dann nämlich vergrößert er oder sie das Feld der Politik, das von ihm/ihr bzw. wieder auszuführen ist. Aufgrund dieses Dilemmas stellen sich letztlich zwei Alternativen: Eine Art >Dienst nach Vorschriftı, bei dem man in möglichst wenig Kompetenzprobleme gerät - oder aber umgekehrt die Übernahme von Verantwortung, im vollen Bewusstsein, genau deswegen negative Konsequenzen befürchten zu müssen. Morstein Marx plädiert für die zweite Lösung.

Manches an dem, was Morstein Marx beschreibt, erinnert auf den ersten Blick an das, was Niklas Luhmann mit dem Konzept »brauchbarer Illegalität« beschreibt. Luhmann hat dieses Konzept in seiner Schrift »Funktionen und Folgen formaler Organisationen « entwickelt, dessen Vorwort wiederum von Fritz Morstein Marx stammt. ${ }^{20}$ Was Morstein Marx' Ansatz von Luhmanns unterscheidet, ist weniger die Diagnose als die Lösung: Morstein Marx' Blick ist auf den Verwaltungsmitarbeiter gerichtet, der sich zwischen "Stehen und Fallen« (Neuntes Kapitel) gegen die Vorgaben der Politik, gegebenenfalls auch seines eigenen Amtsleiters, behaupten muss, da er sich (nicht zuletzt in seinem Amtseid) auf die Wahrung des Gemeinwohls verpflichtet hat. Gemeinsam ist ihnen der Blick auf das Gelingen des Unwahrscheinlichen, nämlich des Verwaltens (dazu unten der Abschnitt "Bleibendes«). Während jedoch Luhmann das System als Garant dieser Stabilisierungsleistung ansieht (durch die formalisierte Verantwortungsübernahme als Unsicherheitsabsorption ${ }^{21}$ ), so sind diese Garanten bei Morstein Marx der einzelne Verwaltungsmitarbeiter und sein Beamtenethos.

\section{»Ethos« oder »Gefühle»}

Morstein Marx' Lösung des "Dilemmas des Verwaltungsmannes« heißt daher auch: das "Pflichtgefühl 22 $^{22}$ beziehungsweise der 
»Verantwortungssinn «. ${ }^{23}$ Morstein Marx beschäftigte sich zu dieser Zeit ohnehin stark mit dem Amtsethos, ${ }^{24}$ auch in einer produktiven Auseinandersetzung mit seinem Speyerer rechtsphilosophischen Kollegen Hans Ryffel, ${ }^{25}$ der ursprünglich aus der Schweiz stammte.

Während bei Morstein Marx der Amtsethos grundsätzlich positiv konnotiert ist und dem Beamten gleichsam Halt auf schwankendem Grund zu verleihen vermag, bezeichnet das Wort "Gefühl« bei ihm nicht unbedingt Positives. Es wird im vorliegenden Buch immer dann verwandt, wenn es sich um das handelt, was Max Weber mit »ira" (Zorn) bezeichnet, von dem sich der Beamte freizumachen habe. ${ }^{26}$ Gefühle gehören für Morstein Marx nicht in den Bereich der Verwaltung, sondern in den Bereich der Politik beziehungsweise denjenigen der Gesellschaft. Dem »Verwaltungsmann« können sie eher »in den Weg geraten«, wenn er sich um das kümmert, dem er sich verpflichtet habe: dem "Interesse des Publikums, der Gesamtheit, des Volks«. ${ }^{27}$ Der Weg aus diesem Konflikt heißt erneut: „das moralische Fundament des öffentlichen Dienstes«, der »Geist der Widmung «. ${ }^{28}$

Anders als Max Weber, dessen Sympathien eher dem (Verantwortung übernehmenden) Berufspolitiker galten als dem Beamten, dessen höchste Kunst diejenige der ausführenden "Selbstverleugnung «" 29 sei, ${ }^{30}$ ist Morstein Marx' Augenmerk auf den "Verwaltungsmann" gerichtet: Wie gelingt es ihm, mit den widerstreitenden Handlungsanforderungen "Selbsterhaltung im Apparat, Weiterkommen auf der Berufsleiter und Treuhänderschaft in der Erfüllung öffentlicher Aufgaben ${ }^{31}$ zurechtzukommen? Und wie kann die Verwaltung - statt auf den »Peitschenknall des Disziplinarrechts « ${ }^{32} \mathrm{zu}$ setzen - die geeigneten Persönlichkeiten finden, die Interesse am Gemeinwohl haben, und wie kann sie dieses Interesse weiter fördern? Das ist unter anderem das Thema des Dritten Kapitels des "Dilemmas des Verwaltungsmannes«.

Der »Verwaltungsmann" wird hier gezeichnet als eine Art postheroischer Held des Alltags, ${ }^{33}$ der die Kunst vollbringen muss, die politischen Vorgaben umzusetzen und zugleich eine Art Feinjustierung an diesen vorzunehmen, sodass sie weiterhin mit dem zu vereinbaren sind, was der Beamte als das Beste für die Gesamtheit empfindet. Morstein Marx setzt dabei auf einen gut ausgebildeten Generalisten, der sich aber durchaus mit den Problemen ıvor Ort` auskennt:

Verwaltung ist ein an einen bestimmten

Ort gebundenes Unternehmen. Es ist kein

Wandergewerbe. [...] Es muss Personal geben, das für sich in Anspruch nimmt, den Geschäftsgang zu kennen, bestimmte Akten aufzutreiben und laufende Sachen weiterzuleiten, so wie das die besonderen Umstände der Bürokratie verlangen. ${ }^{34}$

Verfahren und Organisation spielen eine große Rolle, »um Kontrolle $\mathrm{zu}$ erzielen und Verantwortlichkeit $\mathrm{zu}$ verwirklichen «. ${ }^{35}$ Verantwortlichkeit bzw. Verantwortungsübernahme sind für Morstein Marx zentrale Begriffe. Ähnlich wie Luhmann fasziniert auch Morstein Marx an der Verwaltung das Wahrscheinlichwerden des Unwahrscheinlichen:

Unsere Untersuchung mag sogar für zimperliche Gemüter den Eindruck erwecken, es werde ihnen hier zugemutet, das Gruseln zu erlernen. Ist es nicht wahr, dass der Nachdruck oft eher auf den Kräften der Disorganisation zu ruhen schien? Wie kommt es dann dazu, daß die Verwaltung unter solchen Bedingungen überhaupt funktioniert? Wie kann überhaupt etwas erreicht werden $?^{36}$

Die Lösung sieht Morstein Marx vor allem in dem bereits beschriebenen Amtsethos, einer Mischung aus Alltags- und Berufsethik. Er spricht insoweit vom "Sinn des Verwaltungsmilieus «. ${ }^{37}$ An dieser Stelle macht sich Morstein Marx psychologische und vor allem soziologische Ansätze nutzbar, die sich - nicht nur positiv - mit der "Administrative Class « beschäftigen. ${ }^{38}$ Er grenzt sich aber zugleich wieder von ihnen ab und stellt eher auf die Rolle im "System « Verwaltung ab (das er jedoch immer in Anführungsstrichen schreibt). Die "persönlichen Motivierungen ${ }^{39}$ resultieren aus der Stelle in der Beamtenhierarchie und der Nähe (oder Distanz) zur »Politik«: Der Beamte im Stab hat andere Interessen als derjenige in der Linie, der Abteilungsleiter muss nicht die gleichen Interessen wie der Minister haben, aber auch nicht die gleichen wie ein nachgeordneter Mitarbeiter. Anders als Luhmann sieht Morstein Marx hierin aber keine letztlich unauflösliche Paradoxie, sondern 
formuliert - wie er selbst schreibt - "unter Preisgabe der akademischen Tugend [...] mit zögerndem Schritt" eine »unkonzessionierte[] Therapie « ${ }^{40}$ Diese gehe dahin, dass Verwaltungsmitarbeiter versuchten, "andere zur Beteiligung, zum unterstützenden Selbsteinsatz, zum Engagement « zu bringen. ${ }^{41}$ Wichtig seien dabei auch Symbole $^{42}$ (Morstein Marx hatte nicht umsonst zu Ernst Cassirers $^{43}$ Zeit in Hamburg studiert) und schließlich sogar eine Art (Weber'sche, aber zugleich wohlwollende) Idealtypik eines Beamtentums als Orientierungsmaßstab, also ein »Verständnis des Milieus der Verwaltung - ein eindringendes Verständnis, das sich bloßer Erfahrung nicht vorschnell beugen darf.$^{44}$ Mit diesen Worten endet das "Dilemma des Verwaltungsmannes«.

\section{Biografisches}

"Gefühle» - das Wort hätte Morstein Marx, der es verabscheute, seinen Geburtstag $\mathrm{zu}$ feiern, sicherlich einen Schauer über den Rücken gejagt. Warum war das so?

Dieses ging sicherlich auf seine harte Auseinandersetzung mit sich selbst zurück. Er, der sich noch begeistert als Freiwilliger im Ersten Weltkrieg gemeldet hatte und nichts sehnlicher (vergebens) erhofft hatte, als an die Front zu kommen, hatte im sogenannten "Hamburger Rathaussturm» erlebt, wie es ist, dem Tod Auge in Auge gegenüberzustehen. ${ }^{45}$ Während er damals noch auf der Seite der "Bahrenfelder «, einer Art Freikorps aus Hamburger Bürgersöhnen, das Rathaus gegen die zu Recht wegen eines Lebensmittelskandals aufgebrachten weniger begüterten Hamburgerinnen und Hamburger verteidigt hatte, ${ }^{46}$ nabelte sich Fritz Morstein Marx nach und nach von seinem ebenso unkonventionellen wie deutschnationalen Vater ab und stellte sich als Referendar der Rechte und danach als junger Hamburger Regierungsrat ganz in den Dienst der Republik. ${ }^{47} \mathrm{Zu}$ seinen Förderern gehörten neben seinem (durchaus konservativen) Doktorvater Kurt Perels auch Albrecht Mendelssohn Bartholdy, über den er wohl auch mit den Gedanken Ernst Cassirers in Berührung kam, und schließlich Carl Petersen, der als Hamburger Bürgermeister bei der nationalsozialistischen "Machtergreifung « demissionierte. Petersen ist »Das
Als Petersen von seinem Bürgermeisteramt zurücktrat und Perels und Mendelssohn Bartholdy demütigende Fragen $\mathrm{zu}$ ihrer jüdischen Herkunft beantworten mussten (Perels sollte diese Schmach nicht überleben und auch Mendelssohn Bartholdy wurde schwer krank und starb 1936), ${ }^{49}$ entschied sich Morstein Marx zur Emigration in die USA. Von dort aus sollte er, US-amerikanischer Staatsbürger, Wissenschaftler und Verwaltungspraktiker, zum Wintersemester 1962/63 an die Hochschule für Verwaltungswissenschaften in Speyer berufen werden. Auf die einzelnen Umstände und auf Morstein Marx' beruflichen und wissenschaftlichen Weg zwischen 1933 und 1962 kann an dieser Stelle nicht näher eingegangen werden. ${ }^{50}$ Es sei nur darauf hingewiesen, dass Carl Joachim Friedrich, seit 1956 Inhaber einer Professur in Heidelberg (unter Fortdauer einer Professur in Harvard), vermutlich nicht ganz unwichtig dafür war, dass die Wahl auf Morstein Marx fiel. ${ }^{51}$ Morstein Marx sollte dann auch mit seiner Familie in Heidelberg wohnen und die für ihn nicht untypischen anstrengenden Touren per Bus (und einmal jährlich auch zu Fuß) nach Speyer unternehmen. Er forderte - dem Ideal seines ethisch handelnden "Verwaltungsmannes" verpflichtet - sein Leben lang sich (und vor allem den ihm nahestehenden Personen) Enormes ab, bis er im Oktober 1969 nach einem Kletterunfall verstarb.

\section{Bleibendes}

Warum sollte man das "Dilemma des Verwaltungsmannes« wieder einmal lesen?

Zum einen, weil es unglaubliche Freude bereitet. Wir lernen hier einen Autor kennen, der mit viel Liebe und ebenso viel Humor verwaltungsanthropologische Vignetten wie diejenige vom Beamten als Wärter eines Goldfischglases zeichnet, an dem der Beamte geduldig Algen züchte, ${ }^{52}$ um Transparenzanforderungen entgegenzuwirken.

Morstein Marx, der seine theoretischen Grundlagen zumindest in seinen späten Jahren eher verschleiert, lässt sich aber keinesfalls auf die Rolle eines Verwaltungshumoristen reduzieren. Dagegen spricht nicht nur die Geschichte seiner Emigration aus Gewissensgründen im Jahr 1933 und seiner Remigration. Wie auch in seinen persönlichen 
Handlungsmaximen geht es Morstein Marx insgesamt um einen aufklärerischen Appell hinsichtlich einer demokratisch gesinnten Verwaltung, die als Corps durch einen hohen Verwaltungsethos zusammenzuhalten sei. Entgegenzuwirken sei hingegen der Tendenz, dass "im Zeitalter der funktionalen Expertise die Verwaltung häufig durch die Vielheit der Stimmen der Spezialisten übertönt wird, die sich in ihre eigenen Dinge verbeißen, ohne sich einer allgemein orientierten Verwaltwortlichkeit unterwerfen $\mathrm{zu}$ wollen «. ${ }^{33}$ Morstein Marx' Appell an ein "Beamtentum, das sich als Ganzes verstehen « könne und nur so "zu einer wahren Dienstgesinnung fähig " sei, ${ }^{54}$ ist alles andere als sleichte

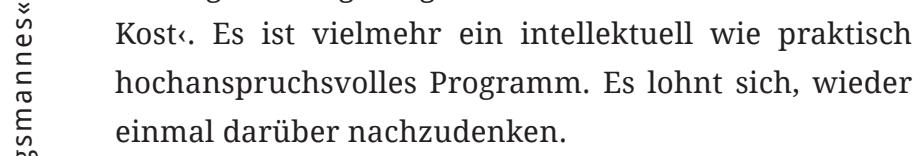


1 Klaus König: »Morstein Marx, Fritz«, in: Neue Deutsche Biographie 18 (1997), S. 159

2 Margrit Seckelmann: »urist im Porträt: Fritz Morstein Marx (19001969) - Inspirator einer svergleichenden Verwaltungswissenschaft «", in: Recht und Politik. Zeitschrift für deutsche und europäische Rechtspolitik 2/2017, S. 207-215, hier S. 211.

3 Margrit Seckelmann: »Die Geburt der Verwaltungswissenschaft aus dem Geiste der Demokratie. Fritz Morstein Marx (1900-1969)«, in: Carsten Kremer (Hg.): Die Verwaltungsrechtswissenschaft der frühen Bundesrepublik (1949-1977), Tübingen 2017, S. 89-105, 89f.

4 Fritz Morstein Marx: Das Dilemma des Verwaltungsmannes, Berlin 1965; S. 7: »Dies Buch handelt vom Verwalten.«

5 Fritz Morstein Marx: »Freiheit und Bürokratie. Über die Natur des Amtsschimmels«, in: Archiv für Rechts- und Sozialphilosophie 42 (1956), S. 351-382.

6 Niklas Luhmann: Der neue Chef, hg. von Jürgen Kaube, Berlin 2016; S. 7: „Der Wechsel des Vorgesetzten gehört zu den wenigen aufregenden Ereignissen im Verwaltungsalltag."

7 Zum (Nicht-)Verhältnis von Morstein Marx und Luhmann vgl. Seckelmann: »Die Geburt der Verwaltungswissenschaft", S. 98-99.

8 Ein Hinweis auf drei solche Aufsätze als Vorläufer findet sich bei Morstein Marx: Das Dilemma des Verwaltungsmannes, S. 8; leider werden die fraglichen Aufsätze aber weder dort noch im Literaturverzeichnis genau bezeichnet. Es handelt sich vermutlich um: Fritz Morstein Marx: "The Mind of the Career Man«, in: Public Administration Review 20 (1960), S. 133-138, ders.: "Control and Responsibility in Administration: Comparative Aspects«, in: Ferrel Heady / Sybil Stokes (Hg.): Papers in Comparative Public Administration. Ann Arbor 1962, S. 145-162; und ders.: »The Higher Civil Service as an Action Group in Western Political Development", in: Joseph La Polombara (Hg.): Bureaucracy and Political Development, Princeton 1963.

9 Morstein Marx: Das Dilemma des Verwaltungsmannes, S. 7.

10 Ebd., S. 7f.

11 Ebd., S. 8.

12 Gabriel Almond / Sidney Verba: The Civic Culture. Political Attitudes and Democracy in Five Nations, Princeton 1963; dazu u. a. Bettina Westle: »Politische Kultur», in: Hans-Joachim Lauth (Hg.): Vergleichende Regierungslehre. Eine Einführung, Wiesbaden 2002, S. 319-341, insbesondere S. 319-322.

13 Vgl. etwa Luther Gulick: "Notes on the Theory of Organization «, in: ders. / Lyndall F. Urwick (Hg.): Papers on the Science of Administration, New York 1937, S. 1-45.

14 Seckelmann: "Jurist im Porträt«, S. 214.

$15 \mathrm{Vgl}$. insbesondere Herbert A. Simon: "The Proverbs of Administration«, in: Public Administration Review 6 (1946), S. 5367.

16 Stephan Kirste: "Carl Joachim Friedrich (1901-1984)«, in: Peter Häberle / Michael Kilian / Heinrich Amadeus Wolff ( $\mathrm{Hg}$.): Staatsrechtslehrer des 20. Jahrhunderts. Deutschland - Österreich - Schweiz, Berlin 2015, S. 555-572.

17 Jörg Bogumil/WernerJann:Verwaltung und Verwaltungswissenschaft in Deutschland. Wiesbaden ${ }^{2} 2009$, S. 39-41.

18 Woodrow Wilson: "The Study of Administration«, in: Political Science Quarterly 2 (1887), S. 197-222.

19 Herbert A. Simon: Administrative Behavior. A Study of DecisionMaking Processes in Administrative Organizations, New York 1947, 241.

20 Niklas Luhmann: Funktionen und Folgen formaler Organisationen, Berlin ${ }^{4} 1995$, S. 304-314

21 Niklas Luhmann: Verantwortung und Verantwortlichkeit. Vortrag am 1. 12. 1961 in Speyer, wieder abgedruckt in: ders.: Schriften zur Organisation. Bd. 1: Die Wirklichkeit der Organisation, hg. von Veronika Tacke / Ernst Lukas, Wiesbaden 2018, S. 47-58, hier S. 55.

22 Morstein Marx: Das Dilemma des Verwaltungsmannes, S. 68.

23 Ebd., S. 92.

24 Fritz Morstein Marx: »Beamtenethos und Verwaltungsethik«, in: Verwaltungsarchiv 54 (1963), S. 323-344.

25 Hans Ryffel: »Eigenverantwortlichkeit«, in: Fritz Morstein Marx (Hg): Verwaltung. Eine einführende Darstellung, Berlin 1965, S. 456-471.

26 Max Weber: „Politik als Beruf, Zweiter Vortrag im Rahmen einer Vortragsreihe ,Geistige Arbeit als Beruf«", gehalten im Revolutionswinter 1918/1919 vor dem Freistudententischen Bund in München, wieder abgedruckt in: Johannes Winckelmann (Hg.): Gesammelte politische Schriften, Tübingen 1971, S. 505-560, hier S. 512.

27 Morstein Marx: Das Dilemma des Verwaltungsmannes, S. 69

28 Ebd.

29 Weber: Politik als Beruf, S. 512.

30 Etwas zuspitzend formuliert. Genaueres zum Verhältnis Morstein Marx' zu Weber bei Margrit Seckelmann: "Postheroische Verwaltung: Fritz Morstein Marx«, in: dies. / Johannes Platz (Hg.): Remigration und Demokratie in der Bundesrepublik nach 1945. Ordnungsvorstellungen zu Staat und Verwaltung im transatlantischen Transfer, Bielefeld 2017, S. 130-152, hier 146151.

31 Morstein Marx: Das Dilemma des Verwaltungsmannes, S. 70.

32 Ebd.

33 Seckelmann: »Postheroische Verwaltung«, S. 151f.

34 Morstein Marx: Das Dilemma des Verwaltungsmannes, S. 143.

35 Ebd.

36 Ebd., S. 153 (sic!).

37 Ebd., S. 194

38 Harold Stein: "The British Administrator's World», in: Public Administration Review 22 (1962), S. 227-236.

39 Morstein Marx: Das Dilemma des Verwaltungsmannes, S.197.

40 Ebd., S.198.

41 Ebd., 199.

42 Ebd.

43 Ernst Cassirer: Philosophie der symbolischen Formen. 3 Bde., Berlin, 11923-1929.

44 Morstein Marx: Das Dilemma des Verwaltungsmannes, S. 200.

45 Margrit Seckelmann: ")Mit Feuereifer für die öffentliche Verwaltung`. Fritz Morstein Marx - Die frühen Jahre (1900-1933)«, in: Die Öffentliche Verwaltung. Zeitschrift für Öffentliches Recht und Verwaltungswissenschaften 66 (2013), S. 401-415.

46 Dazu ebd. sowie Margrit Seckelmann: "»Mit seltener Objektivität Fritz Morstein Marx - Die mittleren Jahre (1934-1961)«, in: Die Öffentliche Verwaltung. Zeitschrift für Öffentliches Recht und Verwaltungswissenschaften 67 (2014), S. 1029-1048, hier S. $1045 f$.

47 Seckelmann: »Mit Feuereifer für die öffentliche Verwaltung«, S. 413 und 415.

48 Auf dem Vorsatzblatt findet sich gedruckt die Widmung »Dem Andenken an einen aufrechten Mann - Bürgermeister Carl Petersen 1868-1933«.

49 Seckelmann: "Mit Feuereifer für die öffentliche Verwaltung", S. 405-407.

50 Vgl. aber Seckelmann: "Mit seltener Objektivität« sowie dies.: »Mit Verständnis für den ıVerwaltungsmann«: Fritz Morstein Marx - Die späten Jahre (1934-1962)«, in: Die Öffentliche Verwaltung. Zeitschrift für Öffentliches Recht und Verwaltungswissenschaften 70 (2017), S. 649-668. 


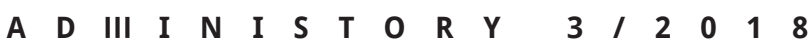

51 Seckelmann: »Mit Verständnis für den ^Verwaltungsmann «, S. 667.

52 Morstein Marx: Das Dilemma des Verwaltungsmannes, S. 66.

53 Ebd., S. 153.

54 Ebd. 


\section{Abstract}

In seiner Schrift Das Dilemma des Verwaltungsmannes von 1965 bringt Fritz Morstein Marx, der Verwaltungswissenschaftler wie Verwaltungspraktiker auf beiden Seiten des Atlantiks tätig war, das Problem des Verwaltens folgendermaßen auf den Punkt: Verwalten muss auch immer eine gestalterische Komponente innehaben. Tut es das nicht, kann eine solche Beschränkung auf den bloßen Normvollzug langfristig zu Politikverdrossenheit führen. Jedoch droht der „Verwaltungsmann" dabei seine Kompetenzen zu überschreiten, weswegen er Nachteile Verkriechen in ein Mauseloch, wo man in möglichst wenig Kompetenzprobleme gerät - oder aber umgekehrt die Übernahme von Verantwortung, auf die Gefahr hin, negative Konsequenzen zu befürchten. Morstein Marx plädiert für die zweite Lösung, bindet sie aber zugleich in ein Beamtenethos ein.

\section{About the Author}

Margrit Seckelmann, Ass. iur. (Berlin), M. A. (Berlin), Dr. iur. (Frankfurt am Main), Dr. habil. (Speyer) studied Law and History in Heidelberg and Berlin. She wrote her LL. D. thesis at the Max-Planck-Institute of European Legal History in Frankfurt am Main. Her habilitation at the German University for Administrative Sciences in Speyer has recently been published (Evaluation und Recht. Strukturen, Prozesse und Legitimationsfragen staatlicher Wissensgewinnung durch (Wissenschafts-)Evaluationen, Tuebingen: Mohr Siebeck 2018). She works as head of administration and as a researcher at the German Research Institute for Public Administration. She has published widely on the history and theory of public administration, mainly on the history of administrative ideas. 